\title{
Strategy implementation for the CTA Atmospheric monitoring program
}

\author{
Michele Doro ${ }^{1, a}$, Michael Daniel ${ }^{2}$, Raquel de los Reyes ${ }^{3}$, Markus Gaug $^{4}$, and Maria Concetta Maccarone ${ }^{5}$ for the CTA \\ Consortium \\ ${ }^{1}$ University and INFN Padova, via Marzolo 8, 35131 Padova, Italy \\ 2 Department of Physics, University of Liverpool, Liverpool L69 7ZE, UK \\ ${ }^{3}$ Max-Planck-Institut fuër Kernphysik, PO Box 103980, 69029 Heidelberg, Germany \\ ${ }^{4}$ Department of Physics, Universitat Autónoma de Barcelona, Campus UAB, 08193 Bellaterra, Spain \\ ${ }^{5}$ Istituto di Astrofisica Spaziale e Fisica Cosmica di Palermo, IASF-Pa/INAF, via Ugo La Malfa 153, \\ 90146 Palermo, Italy
}

\begin{abstract}
The Cherenkov Telescope Array (CTA) is the next generation facility of Imaging Atmospheric Cherenkov Telescopes. It reaches unprecedented sensitivity and energy resolution in very-high-energy gamma-ray astronomy. CTA detects Cherenkov light emitted within an atmospheric shower of particles initiated by cosmic-gamma rays or cosmic rays entering the Earth's atmosphere. From the combination of images the Cherenkov light produces in the telescopes, one is able to infer the primary particle energy and direction. A correct energy estimation can be thus performed only if the local atmosphere is well characterized. The atmosphere not only affects the shower development itself, but also the Cherenkov photon transmission from the emission point in the particle shower, at about 10-20 km above the ground, to the detector. Cherenkov light on the ground is peaked in the UV-blue region, and therefore molecular and aerosol extinction phenomena are important. The goal of CTA is to control systematics in energy reconstruction to better than $10 \%$. For this reason, a careful and continuous monitoring and characterization of the atmosphere is required. In addition, CTA will be operated as an observatory, with data made public along with appropriate analysis tools. High-level data quality can only be ensured if the atmospheric properties are consistently and continuously taken into account. In this contribution, we concentrate on discussing the implementation strategy for the various atmospheric monitoring instruments currently under discussion in CTA. These includes Raman lidars and ceilometers, stellar photometers and others available both from commercial providers and public research centers.
\end{abstract}

\section{Introduction}

Currently in its design stage, the Cherenkov Telescope Array (CTA, see Fig. 1) is an advanced facility for groundbased very-high-energy gamma-ray astronomy [1,2]. It is an initiative to build the next-generation ground-based very-high-energy gamma ray observatory covering the energy range from a few tens of $\mathrm{GeV}$ up to over a hundred $\mathrm{TeV}$ with unprecedented sensitivity. The design of CTA is based on currently available technologies and builds upon the success of the present generation of groundbased Cherenkov telescope arrays (H.E.S.S., MAGIC and VERITAS $^{1}$ ).

Nowadays, the main contribution to the systematic uncertainties of imaging Cherenkov telescopes stems from the uncertainty in the height- and wavelength-dependent atmospheric optical properties for a given run of data. Atmospheric quality affects the measured Cherenkov yield in several ways: the air-shower development and Cherenkov light production, depending on the molecular density profile, the loss of photons due to scattering and absorption of Cherenkov light out of the camera fieldof-view, resulting in dimmer images, and the scattering of photons into the camera, resulting in blurred images. Despite the fact that several supplementary instruments are currently used to measure the atmospheric transparency

\footnotetext{
a e-mail: michele.doro@pd.infn.it

1 www.mpi-hd.mpg.de/hfm/HESS/; magic.mpp.mpg.de; veritas.sao.arizona.edu
}

together with Cherenkov telescopes, their data are mainly used to retain good-quality observation time slots, and only a minor effort has been made to routinely correct data with atmospheric information [3-6]. This situation will improve (MAGIC and H.E.S.S. are already characterizing atmosphere to some extent, albeit not yet in a standard way and not properly tested) with the CTA atmospheric monitoring and calibration program $[7,8]$. There are several goals behind this program. The first is to increase the precision and accuracy in the energy and flux reconstruction through the use of one or more atmospheric instruments. Secondly, a precise and continuous monitoring of the atmosphere will allow for an increase of the telescope duty cycle with the extension of the observation time during hazy atmospheric conditions, which are normally discarded in the current experiments because of the uncertainty in the data reconstruction. Finally, a possible "smart scheduling", i.e. an adaptation - if required - of the observation strategy during the night that considers the actual atmospheric condition can be activated with a precise monitoring program of the atmospheric conditions.

This contribution is structured as follows. In Sect. 2 we briefly discuss the influence of the atmosphere on IACT (Imaging Atmospheric Cherenkov Telescope) data. In Sect. 3 we summarize the development activities for the instrumentation and methods for the atmospheric monitoring program in CTA. In Sect. 4 we discuss the main tasks required to the program as well as the

This is an Open Access article distributed under the terms of the Creative Commons Attribution License 4.0, which permits unrestricted use, distribution, and reproduction in any medium, provided the original work is properly cited. 


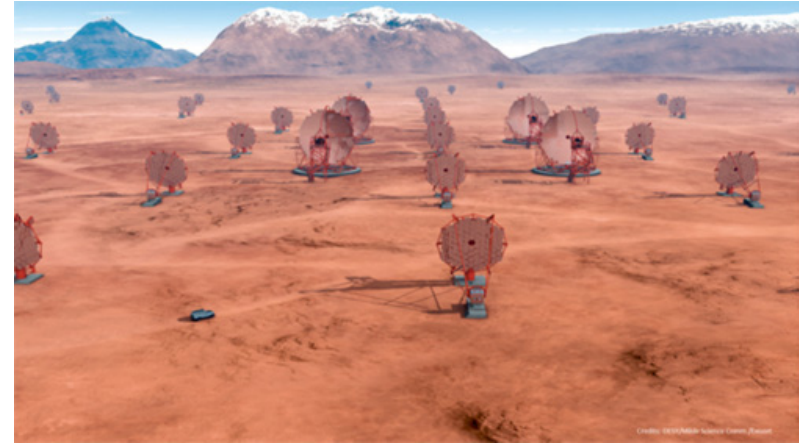

Figure 1. Artistic sketch of one of the two CTA sites. Several telescopes of different sizes will be deployed.

implementation strategy. In Sect. 5 we discuss the possible usefulness of having interchange of data between CTA and ground-based and satellite atmospheric monitoring networks. We end with a summary.

\section{The influence of the atmosphere on IACT data}

Although IACTs are normally placed at astronomical sites, characterized by extremely good atmospheric conditions, the local atmosphere is potentially influenced by phenomena occurring at tens to thousands of kilometers away, and thus should be continuously monitored. While the molecular content of the atmosphere varies very slowly at a given location during the year, and slowly from place to place, aerosol concentrations can vary on time-scales of minutes and travel large, inter-continental, distances. Most of them are concentrated within the first $3 \mathrm{~km}$ of the troposphere, with the free troposphere above being orders of magnitude cleaner. Aerosol sizes reach from molecular dimensions to millimeters, and the particles remain in the troposphere from 10 days to 3 weeks. The sizes are strongly dependent on relative humidity. Different types of aerosol show characteristic size distributions, and an astronomical site will always show a mixture of types, with one possibly dominant type at any given time and/or altitude. Light scattering and absorption by aerosols needs to be described by Mie theory or further developments of it, including non-sphericity of the scatterer. Aerosols generally have larger indexes of refraction than that of water, and some show also a small imaginary part. Contrary to the typical $\lambda^{-4}$ wavelength dependency of Rayleigh-scattering molecules, aerosols show power-law indexes (the so-called Angström coefficients) from 0 to 1.5, i.e. a much weaker dependency on wavelength.

In order to estimate the effect of different atmospheric conditions on the image analysis of IACTs, we have simulated different molecular and aerosol profiles for the MAGIC system, consisting of two telescopes $[10,11]$. Several aerosol scenarios were simulated: $i$ ) enhancements of the ground layer from a quasi aerosol-free case up to a thick layer which reduces optical transmission by $70 \%$, ii) a cloud layer at the altitudes of $6 \mathrm{~km}, 10 \mathrm{~km}$ (cirrus) and $14 \mathrm{~km}$ (volcano debris) a.s.l., and iii) a $6 \mathrm{~km}$ cloud layer with varying aerosol densities. The main results can be summarized in three points:

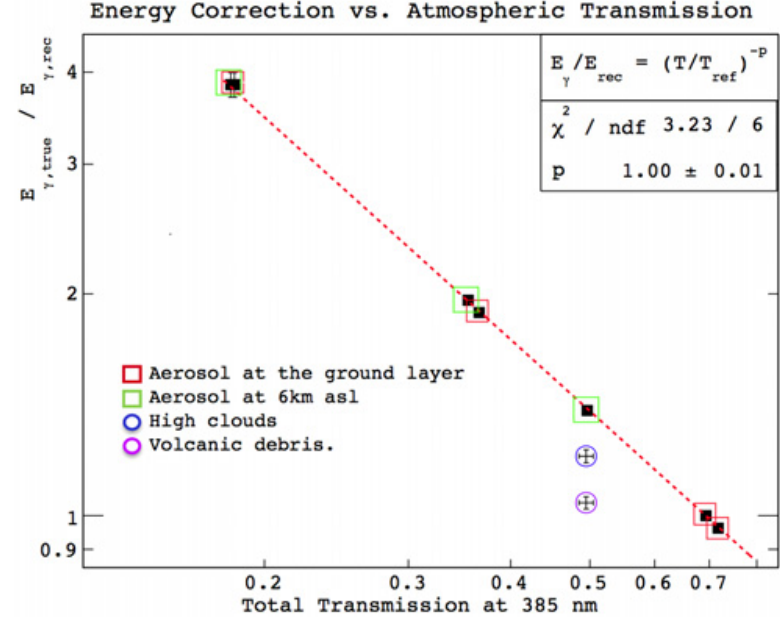

Figure 2. Energy correction factor as a function of the total atmospheric transmission at $385 \mathrm{~nm}$. The red and green points show enhancements of the ground layer and low clouds, respectively, while the pink and dark green symbols refer to high clouds and volcanic debris. The linear dependence is valid only in the case of aerosol over-densities at low heights. $E_{\text {rec }}$ is the reconstructed energy from an initial Monte Carlo event with energy $E_{\gamma} . T_{\text {ref }}$ is a scale transmission at $385 \mathrm{~nm}$ and $-p$ the chisquare optimized index, $T$ is the transmission at $385 \mathrm{~nm}$ for each of the points in the plot. See text for additional details. Taken from [10].

1. Using adapted Monte Carlo simulation, energy and flux can be reconstructed right away, at only the expense of a larger energy threshold, which can be explained by the fact that the faint images of lower energy showers are absent with hazy atmospheres as fewer photons reach the ground. At energies above the new threshold, images observed under large impact distances, i.e. from coming from the shower halo, are gradually lost, and a small degradation of the effective area is obtained;

2. In the case that the aerosol over-density or cloud is below the electromagnetic shower, a simple correction method can be used to restore correct energy and flux reconstruction with the simple use of standard Monte Carlo;

3. When the cloud or aerosol layer is at the shower development region or above, the total extinction is no longer a useful parameter;

The last two points are shown in Fig. 2 where the energy correction is shown as a function of the total transmission at $385 \mathrm{~nm}$. One can see that in the case that the aerosol over-density is located close to the ground, i.e. below the electromagnetic shower development, then the associated correction for the energy reconstruction is precisely obtained from the total transmission, while if the over-density is above, then correlation is broken and height-resolving instruments are required. This is the main motivation for the need of a Raman lidar instrument for CTA. The main findings of this study must be also valid for CTA, albeit the critical height of aerosol layers for the simple linear energy correction to work out must lie considerably lower, since showers of $\mathrm{TeV}$ energies penetrate further down the atmosphere. Previous studies 
have been made $[3,4,12]$ for H.E.S.S. and for the MAGIC mono system, however only for an increase of low-altitude aerosol densities, and in [13] for a reference configuration of CTA, claiming a change in the spectral power-law index of gamma-ray fluxes, when atmospheric aerosol layers are present. In our work, a very small dependence of the spectral index on the aerosol densities could be obtained only with very dense layers. See $[10,11]$ for further details.

\section{Instruments and methods for the CTA atmospheric monitoring program}

\subsection{Raman lidars}

Several institutes in CTA are currently designing Raman lidar systems: the Institut de Fisica d'Altes Energies (IFAE) and the Universitat Autònoma de Barcelona (UAB), located in Barcelona (Spain), the LUPM (Laboratoire Univers et Particules de Montpellier) in Montpellier (France) and the CEILAP (Centro de Investigaciones Laser y sus Aplicaciones) group in Villa Martelli (Argentina). The different groups are designing independently prototypes with different mechanical, optical and steering solutions. Generically, these lidars are characterized by large reflectors of $\geq 1.5 \mathrm{~m}$ diameter, powerful Nd:YAG lasers operated at $2-3$ wavelength (1054 nm and its first and second harmonics), and an optical readout system based on at least 4 channels (355 and $532 \mathrm{~nm}$ elastic and the corresponding two Raman lines). They are steerable and operated mainly at night. More details of those devices can be found in [14].

In addition, two other groups have shown interest in discussing new designs for Raman lidars, one in Adelaide (Australia) that may provide a powerful wind-lidar, and one from INFN (Italy) that will provide a smaller wellcalibrated Raman lidar for instrumental cross-check and site climatology characterization [17].

\subsection{Ceilometer}

A ceilometer is a device primarily used in the aviation industry for cloud reporting and/or measuring the vertical visibility in harsh conditions. The instrument makes use of the lidar technique, using low power infra-red pulsed diode lasers at high pulsing rates to provide eye-safe, reliable, fully automatic $24 / 7$ operation in all weather conditions. The continuous operation of a ceilometer would not disturb the operation of the CTA. Cloud reporting range up to $13 \mathrm{~km}(43,000 \mathrm{ft})$ and backscatter profiling over a full measurement range up to $15 \mathrm{~km}(49,200 \mathrm{ft})$ is possible with a Vaisala CL51 model ceilometer. Advanced single-lens design provides excellent performance even at low altitudes. In favourable conditions the backscattering profile can also be used to monitor boundary layer structures [18].

\subsection{All-Sky Camera}

The All-Sky Camera (ASC) is a passive non-invasive imaging system for night sky atmosphere monitoring. The operation of the ASC would not affect the measurement procedure of CTA. The ASC will determine clouds and an overall atmospheric quality on the short term. In case of a partly cloudy night sky, the cameras will identify the uncovered regions of the sky, which would help to define those regions of the sky where sources can be observed without performance degradation [19].

\subsection{UVscope}

The UVscope [20] is a portable multi-pixel photon detector, NIST-calibrated in the lab, developed at INAF IASF-Palermo (Italy) to support experimental activities in the high-energy astrophysics and cosmic-ray field. The instrument, working in single photon-counting mode, is designed to directly measure light flux in the wavelength range $300-650 \mathrm{~nm}$. Thanks to its features and operational flexibility, the instrument can be used in a wide range of applications. Currently, its primary application is in the framework of the absolute calibration of the ASTRI SST2M telescope prototype [21]. For CTA, the UVscope could be used to study the atmospheric transparency.

The atmospheric transparency can be evaluated by measuring the absorption of the flux of a star, as a function of the air mass traversed, which is related to the star altitude. The measurements of atmospheric transparency are performed with the UVscope by following a star during its path in the sky (tracking mode); in this way it is possible to measure its luminosity as a function of the altitude, from which the sky transparency can be derived. The UVscope can also measure the diffuse emission of the night sky background, NSB, in the same field of view of a given telescope, as successfully performed at the Pierre Auger Observatory during a several years campaign [22]. In the CTA framework, UVscope would follow the same pointing of a given CTA telescope or group of telescopes.

\subsection{Photometric Robotic Atmospheric Monitor}

The F/(Ph)otometric Robotic Atmospheric Monitor (FRAM) is a wide field astronomical imaging camera on a robotic mount to monitor stars in the whole field of view of the Cherenkov telescopes. From the measured light flux of the stars we can determine the atmospheric extinction in this direction with high spatial and temporal resolution without interfering with the Cherenkov observations. The FRAM was successfully operated at the Pierre Auger Observatory [23] and is currently being adapted to CTA. It consists of a commercial robotic astronomical mount, a large-format CCD camera, a photographic telephoto lens, a focuser for the lens, such as the Rigel nStep focuser which mechanically couples to the manual focus wheel of the lens.

While there are many probes capable of monitoring the state of the atmosphere, stars have the advantage of being non-invasive, always available and well understood. The FRAM samples atmospheric properties with high precision at different directions using well-characterized stellar fields and extrapolates these data assuming spatial and temporal uniformity of the atmospheric conditions. 
Secondly, it may perform detailed follow-up observations covering wider, but still limited, areas of the sky immediately after detection of particularly interesting air showers. The measurements of the first kind are carried out using a narrow-field camera mounted full $30-\mathrm{cm}$ astronomical telescope (which is also used for other observations, such as GRB follow-ups), while those of the second kind use a wide-field camera connected to a photographic lens.

\subsection{Molecular profile}

The molecular profile is measured by radio sondes, but can be approximated by global atmospheric data assimilation system, like the GDAS [24]. Its precision depends basically on the proximity of standard radio sonde launch sites, like airports. As soon as the sites for the CTA are selected, we will start a radio sonde campaign of our own to cross-validate the local GDAS data and, upon success, use these data for the (time-dependent) determination of the molecular profile.

\subsection{The Cherenkov Transparency Coefficient method}

Besides any other atmospheric monitoring devices placed on the observatory site, the Cherenkov Transparency Coefficient (CTC) will provide a way to estimate the atmospheric transparency based on the information of the Cherenkov data only. This coefficient has the advantage that is calculated using only observables and calibration parameters (trigger rates, muon efficiency and mean camera gain) from the Cherenkov data taken with the IACTs. The CTC is derived under the assumption that the zenith-corrected single telescope trigger rate is dominated by cosmic-ray protons. Therefore the trigger energy threshold of the telescope $\left(E_{0}\right)$ is inversely proportional to the average pixel gain $g$, the optical throughput of the telescope, parameterized by the muon efficiency $\mu$ and the atmospheric transparency, parameterized by a factor $\eta$ so that $E_{0} \propto(\eta \cdot \mu \cdot g)^{-1}$. Random fluctuations in the trigger of a single telescope are removed by selecting only the read-out events for which at least 2 telescopes are triggered in coincidence. This read-out trigger will therefore depend on the number of active telescopes so the averaged trigger over all $N$ active telescopes is calculated and rescaled by a factor of $k_{N}$ that depends on the telescope multiplicity.

Different correlation studies have been carried out between the CTC and independent measurements from diverse atmospheric monitoring instruments [15]. A large correlation factor of $\sim 0.85$ at blue wavelengths $(\lambda=$ $443 \mathrm{~nm}$ ) has been found between the aerosol optical depth measurements performed by the MISR satellite and the CTC. The same order of magnitude has been found in the correlation with lidar and Radiometer systems placed on the H.E.S.S. site. It does not (yet) provide information about the height profile of the atmospheric disturbances, which would be required for a full data correction, though. These positive correlation results prove the capability of this coefficient to characterize the aerosol optical depth affecting the Cherenkov observations. Since the CTC is extracted from the Cherenkov data used in the IACT analysis, it is easy to study the relation between the atmosphere quality and the systematic errors introduced in the spectral analysis results due to a bad calibration of the atmosphere [15]. Therefore, the CTC is currently used in H.E.S.S. as a data quality selection criteria, establishing a criteria for the maximum allowed systematic error in the spectral analysis results of $\sim 20 \%$. The relation of the CTC not only with the Cherenkov analysis systematics but also with independent atmospheric instrumentation on-site opens the possibility of an automatic interaction with a central autoscheduler concerning the atmospheric conditions for observations.

\section{Strategy implementation for CTA Atmospheric Monitoring program}

As discussed above, an ensemble of instruments is foreseen to be installed at the site(s) of CTA for the Atmospheric Monitoring. Obviously, these instruments, when properly characterized and inter-connected, have various uses. In this section, we outline the main tasks we expect from those instruments, as well the strategy implementation.

\subsection{TASK-1. Climatology build-up}

This task deals with studies carried out as soon as the site decision is made, even before the first telescopes are installed. Its outcome will be needed for the decision as to whether data models like GDAS or SENES are reliable enough, and which part can be used to replace direct measurements. During this time, also a profound knowledge of the aerosol mass distributions, their occurrence and variability should be obtained. In addition, we use this phase to gain full knowledge about the correction of the telescope data for atmospheric influences. During this time, all available atmospheric monitoring instruments should be in place, and their interplay with the telescopes be studied, as well as their contribution to the correction factors of the telescope data.

Among the instruments foreseen for this task, we propose as primary $(\mathrm{P})$ and secondary $(\mathrm{S})$ - in terms of relevance -:

- (P) Commercial weather stations, satellites and local historical data, national radars, radio sondes, INFN Raman lidar, ASC.

- (S) Ceilometers, All-sky Camera, UVscope and FRAM, Raman lidars.

Commercial weather stations are meant to generally characterize the current situation of the site in terms of humidity, temperature, wind speed and direction and pressure and correlations of rain fall and thunderstorm activity with the previous parameters. However, the collection of multi-annual data and their correlation with available satellite data, historical data and with data from possible closeby national radar can reinforce the knowledge of long-term behavior at the site, and therefore 
allow to better model any current situation and allow to make predictions. These instruments and methods should therefore be installed/implemented at the site as soon as possible. In addition, the use of a sun photometer inside global networks like AERONET, will allow not only to validate and disseminate our data, but to have a more precise global scenario. Finally, radiosondes are extremely important in the first years to not only monitor the situation in-situ, but also collect valuable information of the atmospheric vertical structure. The launches of balloons are only foreseen for the first years of CTA, probably before the start of operation. The INFN Raman lidar comes from the ARCADE project [25] and is a wellcalibrated lidar. It has a $20 \mathrm{~cm}$ diameter mirror and is more easily portable than other Raman lidars under development for CTA. It will be brought to the site as soon as it becomes available with the additional task of cross-calibrating the final Raman lidars for the site. The ASC is in a mature stage and will be installed at the site soon.

However, additional instruments can complement the acquisition of a climatology of the site, as well as themselves being better calibrated and commissioned. The ceilometer will allow to retrieve information on the cloud presence and distribution in the sky both day and night. Stellar photometers data like that from the FRAM will allow to commission those devices as well as know the precise extinction in given directions. Finally, Raman lidar data, compared with ASC, FRAM and UVscope data will allow to improve the overall quality and precision of the results.

\subsection{TASK-2. Off-line data selection}

The goal of the CTA atmospheric calibration is to minimize any loss of data due to non-optimal atmospheric conditions during data selection. This shall be achieved through smart scheduling according to the needs of the physics analysis behind the observation, in combination with the available atmospheric conditions in a given pointing direction. If the conditions do not allow such a strategy, then offline data correction or even rejection will gain an important role.

Among the instruments and methods that can be used for such a task are:

- (P) Raman lidars, UVscope and FRAM and CTC.

- (S) All-sky camera.

Raman lidar data will provide spatially resolved information, but the information coming from integrated optical depth from the FRAM and the UVscope will allow to improve the precision. Finally, from the data of the telescope, we can cross-check the above information with the CTC.

Even if the ASC is an all-sky instrument, it also serves the purpose of measuring the integral extinction from a certain direction in the sky and therefore can complement the above information.

\subsection{TASK-3. Off-line data correction}

Data taken during non-optimal atmospheric conditions can be corrected by the use of adapted Monte Carlo simulations or clever algorithms that make use of atmospheric information [6]. As a result, not only the energy and flux can be correctly retrieved for several situations, but also the overall duty cycle of the experiment is increased. This task is similar to Task-2, but requires more precision, as well as more complicated analysis algorithm, interactions with the data reconstruction pipeline, the Monte Carlo simulations, etc.

The instruments and methods for this task are therefore the same as for Task-2,

- (P) Raman lidars, UVscope and FRAM and CTC.

- (S) All-sky camera.

The reason we discuss this task separately is that its complexity is higher and requires the densest data coverage achievable, as well as robustness of the result, in order to reduce and control the final systematics quoted in the scientific publications.

\subsection{TASK-4. On-line smart scheduling}

This section deals with the instrumentation and procedure to assure that the CTA points always to sources under conditions which permit to carry out the relevant physics analysis afterwards. Depending on the altitude and thickness of aerosol/cloud layers, the energy threshold as well as angular and energy resolution may be degraded, until an observation under the given conditions would not make sense any more. Given that CTA will deploy instrumentation that allows to predict these parameters, a smart scheduling program will decide, at any time, to select only observations fulfilling the criteria on threshold and resolution. For this, we therefore work with allsky instruments, rather than instruments that classify the atmosphere in the pointing direction as in Task-2 and -3. Of importance are:

- (P) All-sky Camera, Ceilometer.

- (S) Raman lidars, FRAM, UVscope and CTC.

It is true however, that while the above instruments provide information on the whole sky, the precise knowledge on the current observation is provided by the pointing instruments. For example, Raman lidars can provide a precise estimation on the current energy threshold of the experiment, and in case of peculiar objects this could suggest to move to another source in the sky.

\subsection{TASK-5. Weather now-cast, fore-cast, alerts and protection}

Atmospheric instruments provide online information on the weather and can be used to make short-term forecasts that are useful not only for data-taking, but for planning trips, interventions, etc. Atmospheric instruments can be used to forecast or early detect possible risky situations 
for the experiment, like rain, lightning, thunderstorms, etc., with the possibility to raise alerts and take decisions over subsystems. Of primary importance are:

- (P) Commercial weather stations, national weather radars, satellites, remote rain sensors, lightning sensors, ceilometers.

\section{Networks of atmospheric instruments}

Ground-based atmospheric monitoring networks provide input to global atmospheric models. Networks are important also because they impose standardized algorithms and reduce bias. For a more detailed discussion, we refer the reader to [26].

The biggest atmospheric network for chemical composition of the atmosphere is the Global Atmospheric Watch (GAW) programme of the World Meteorological Organisation (WMO) [27]. Regarding aerosol radiative properties, there are other global networks, like the AErosol RObotic NETwork (AERONET) [28]. Other smaller networks exist like GAW Aerosol LiDAR Observations Network (GALION) and sub-networks as the European Aerosol Research LiDAR NETwork (EARLINET) or the MicroPulse LiDAR NETwork (MPLNET) [29]. Regarding clouds, CLOUDNET monitors the cloud coverage and its vertical structure [30].

Satellites are also available to offer accurate measurements in regions not covered (yet) by groundbased weather stations. Satellites can be geostationary or polar. Satellites typically measure the cloud emissivity in infrared or sunlight scattered by aerosols or clouds, or record backscattered light. In this category, we mention CALIPSO, MODIS GOES [31], among others. Data from satellites are usually available publicly one year later or once the mission has ended and therefore can be used easily to evaluate atmospheric conditions of a site candidate for an astroparticle physics experiment.

It is currently under investigation what kind of interaction the CTA instrumentation can sustain with these networks. From one side, CTA can obviously profit from the wealth of data provided by those networks. On the other side, these networks could be interested in obtaining data from the Atmospheric Monitoring instruments of CTA, especially because these will be located at remote sites, normally not covered by ground-based installations.

\section{Conclusion}

CTA will constitute the leading project in high energy gamma-ray astronomy in the future decades. It can provide excellent data quality provided the atmosphere is well determined during data-taking. To achieve this goal, an ensemble of instruments is currently planned for the CTA sites that can perform pointed and allsky observation with several different tasks including providing a site climatology, data selection and correction, smart scheduling as well as climate forecast and instrument protection.
We gratefully acknowledge support from the agencies and organizations listed under Funding Agencies at http://www.ctaobservatory.org/

\section{References}

[1] M. Actis et al. [CTA Coll.], Exper.Astron. 32 (2011) 193-316.

[2] B.S. Acharya et al. [CTA Coll.], APP 43 (2013) 3-18

[3] S.J. Nolan et al., Procs. 30th ICRC (2007)

[4] D. Dorner et al., A\&A 493 (2009) 721-725

[5] R. De Los Reyes et al., Procs. 33rd ICRC (2013) ID-0610

[6] C. Fruck et al., Procs. of the 33rd ICRC, Rio de Janeiro, Brasil (2013)

[7] M. Doro et al. [CTA Coll.], Procs. 33rd ICRC (2013) ID-0151

[8] M. Gaug et al., [CTA Coll.] Procs. SPIE, Montreal, Canada, Id 9149-45

[9] D. Garrido et al., Procs. of the 33rd ICRC, Rio de Janeiro, Brasil (2013)

[10] D. Garrido. Ph.D. thesis. Univeristat Autónoma Barcelona (2011)

[11] D. Garrido et al., Procs. of the 1st AtmoHEAD Conference, Saclay, France (2013)

[12] K. Bernlöhr, Astrop. Phys. 12 (2000) 255-268

[13] S.J. Nolan et al., Astrop. Phys. 34 (2010) 304-313

[14] M. Doro et al. [CTA Coll.], Procs. of the 1st AtmoHEAD Confernce, Saclay, France (2013)

[15] J. Hahn et al., Astropart. Phys. 54 (2014) 25-32

[16] J. Hahn et al., AIP Conf. Procs 1505 (2012) 721-724

[17] L. Valore [Auger Coll.], [arXiv : 1402 .6186] [astroph.IM]

[18] A.M. Karkkainen et al., Proc. SPIE 3104, 12-17 (1997)

[19] D. Mandat, Procs. of the 1st Atmohead Conference, Saclay, France (2013)

[20] M.C. Maccarone et al., Nuclear Instrum. \& Methods - Section A, vol. 659, Issue 1, pp. 569-578

[21] M.C. Maccarone et al. [ASTRI Coll. \& CTA Coll.], Proc. SPIE, Montreal, Canada (2014). ID 9149-44

[22] A. Segreto [Auger Coll.], Proc. 32nd ICRC Bejing, China (2011)

[23] S. Benzvi et al. [Auger Coll.], [arXiv:0706.1710] [astro-ph]

[24] P. Abreu et al. [Auger Coll.] Astrop. Ph. 25 (2012) 591-607

[25] M. Buscemi et al. [ARCADE Coll.], Procs. of 33rd ICRC (2013)

[26] K. Louedec, [arXiv: 1403. 2977] [astro-ph.IM]

[27] GAW, www.wmo.int/pages/prog/arep/gaw/gaw home_en.html

[28] AERONET, aeronet.gsfc.nasa.gov \& B.N. Holben et al., Rem. Sens. Environ. 66 (1998) 1-16

[29] EARLINET, earlinet.org, ACTRIS, www.actris. net, MPLNET, mplnet.gsfc. nasa.gov

[30] CLOUDNET, www.cloud-net.org

[31] CALIPSO smsc.cnes.fr/CALIPSO/index.htm MODIS modis.gsfc.nasa.gov. GOES www.goes.noaa.gov 